\title{
Análisis de la incorporación de las tecnologías en el aula de ciencias: implicaciones para la formación docente
}

\author{
ANALYSIS OF THE INTEGRATION OF TECHNOLOGY IN THE SCIENCE CLASSROOM: IMPLICATIONS FOR \\ TEACHER TRAINING \\ A ANÁLISE DA INTEGRAÇÃO DA TECNOLOGIA NAS AULAS DE CIÊNCIAS: IMPLICAÇÕES PARA A FORMAÇÃO \\ DE PROFESSORES
}

José Luis Blancas Hernández* / Diana Patricia Rodríguez Pineda**

\begin{abstract}
Resumen
La incorporación de las tecnologías en la educación científica plantea múltiples desafíos; uno de ellos es cómo apoyar a los profesores a encontrar sentido y significado al uso de las mismas. Los procesos de formación y actualización docente bien podrían verse enriquecidos al considerar y reconocer lo que cotidianamente ocurre en el aula de ciencias con el uso de las herramientas tecnológicas. En este sentido, presentamos el análisis de una actividad que implica el uso de tecnologías, proveniente de los materiales didácticos difundidos por la Secretaría de Educación Pública (SEP) de México. La actividad fue implementada en el aula por un profesor de biología -instalado conceptualmente en el constructivismo- y analizada a partir de indicadores descriptivos repartidos en tres ámbitos de estudio (epistemológico, de aprendizaje y tecnológico) y desde diferentes perspectivas teóricas. Los resultados muestran una actividad centrada en una visión empirista de la ciencia, en la promoción de aprendizajes mecanicistas y en un uso técnico de la tecnología. Los hallazgos se discuten en términos de las implicaciones para pensar en el diseño mucho más situado de la formación y actualización docente.
\end{abstract}

Summary

The incorporation of technology in science education poses multiple challenges; one of them is how to support teachers to make sense and meaning to the use thereof. The formation processes and credentials may well be enriched to consider and recognize what routinely happens in the science classroom with the use of technological tools. Here, we present the analysis of an activity that involves the use of technologies, from educational materials distributed by the Secretariat of Public Education (SEP) of Mexico. The activity was implemented by a biology professor -installed conceptually in constructivism and analyzed based on descriptive indicators divided into three areas of study (epistemological, learning and technology) and from different theoretical perspectives in the classroom. The results show an activity centered on an empiricist view of science, in promoting learning mechanistic and technical use of technology. The findings are discussed in terms of implications for thinking about much more located in teacher training and refresher design.

\section{Resumo}

A incorporação da tecnologia na educação científica coloca vários desafios; um deles é a forma de apoiar os professores a fazer sentido e significado para a sua utilização. Os processos de formação e credenciais podem muito bem ser enriquecido a considerar e reconhecer o que rotineiramente acontece na aula de ciências com o uso de ferramentas tecnológicas. Aqui, apresentamos a análise de uma atividade que envolve o uso de tecnologias, de materiais educativos distribuídos pela Secretaria de Educação Pública (SEP) do México. A atividade foi implementado por um professor de biologia -instalado conceitualmente no construtivismo e analisados com base em indicadores descritivos divididas em três áreas de estudo (epistemológica, aprendizagem e tecnologia) e a partir de diferentes perspectivas teóricas em sala de aula. Os resultados mostram uma atividade centrada na visão empirista da ciência, na promoção da aprendizagem utilização mecanicista e técnico de tecnologia. Os resultados são discutidos em termos de implicações para pensar em muito mais localizada na formação de professores e projeto de reciclagem.

\section{Palabras clave}

Actividades con tecnología, práctica docente, concepciones, innovación educativa, formación docente.

\section{Key words}

Activities with technology, teaching practice, conceptions, educational innovation, teacher training.

\section{Palavras chave}

Atividades com a tecnologia, prática de ensino, concepções, inovação educacional, formação de professores.

\section{Fecha de recepción: 24 de septiembre de 2014 / Fecha de aceptación: 31 de octubre de 2014}

* Maestro en Ciencias en la especialidad de Investigaciones Educativas, Centro de Investigación y de Estudios Avanzados (CINVESTAV), México; correo electrónico: jlblanher@gmail.com

** Doctora en Educación, Línea Enseñanza de las Ciencias, Universidad Pedagógica Nacional (UPN), México. Profesora-investigadora del Cuerpo Académico de Educación en Ciencia; correo electrónico: dpineda@upn.mx 


\section{Introducción}

En la educación en ciencias se reconoce que las tecnologías de la información y comunicación (en adelante tecnologías) ofrecen, por su naturaleza y características, la posibilidad de renovar los procesos de enseñanza y aprendizaje de los contenidos científicos (Sanmartí e Izquierdo, 2001). En México, esta visión tiene una fuerte presencia en la retórica pedagógica del currículo oficial de ciencias para educación secundaria, producto de la reforma planteada en 2006; a través de ella, los profesores son convocados a incorporar tecnologías a sus prácticas de enseñanza como una vía privilegiada para la innovación educativa (SEP, 2006, 2006a).

En este contexto, la Secretaría de Educación Pública (SEP) propuso el material educativo Enseñanza de las Ciencias con Tecnología (ECIT), para que los profesores de ciencias utilicen tecnologías en su práctica docente (SEP, 2007). En su fundamentación se sostiene que ECIT "es una herramienta tecnológica estructurada a partir de una propuesta de enseñanza-aprendizaje de corte constructivista" 1 . Desde este enfoque, el material propone a los maestros secuencias didácticas cuyas actividades demandan el uso de distintas tecnologías (internet, simulaciones, sensores, videos, animaciones, etc.) en el tratamiento de los contenidos de biología, física y química.

Las demandas curriculares sobre el uso de tecnologías en la enseñanza, si bien presentan aspiraciones loables y ambiciosas, esperan que los profesores utilicen herramientas tecnológicas que potencialmente son complejas y poderosas. Esto plantea varios desafíos a los profesores, tales como abandonar formas de trabajo fuertemente sedimentadas, modificar sus concepciones y saberes de lo que implica enseñar ciencias y dominar en poco tiempo materiales con los que tienen una total falta de familiaridad. En este sentido, la utilización de tecnologías en la enseñanza de las ciencias demanda de los profesores un amplio y profundo repertorio de conocimientos, estrategias y habilidades profesionales. Sin embargo, las reformas e innovaciones en la forma-

1 Es posible ampliar la información en: http://www.efit-emat.dgme. sep.gob.mx/ecit/ecitmodelos.htm ción y actualización docente han quedado rezagadas en relación con la reforma al currículo oficial de ciencias para educación secundaria.

Cómo apoyar a los profesores de ciencias para que se apropien de forma relevante de las potencialidades de las tecnologías para el diseño y ejecución de actividades didácticas innovadoras, es uno de los retos que actualmente enfrenta la formación docente. Al respecto, consideramos que las tareas y acciones encaminadas a la formación y actualización docente han de integrar, entre otras cosas, los conocimientos y prácticas que los profesores poseen y que son fruto de su historia como sujetos enteros. En este marco, realizamos un estudio con el propósito de describir, cualitativamente, la realización de una actividad de ECIT en el aula a partir de tres ámbitos de indagación y desde diferentes perspectivas teóricas. Con este estudio pretendemos aportar elementos empíricos que permitan trazar caminos para lograr y fundamentar, desde bases acordes a la práctica, la formación y actualización docente, una certera y atinada introducción de las tecnologías en las aulas de ciencias.

La aproximación analítica que adoptamos en este trabajo se centra en las actividades didácticas que se realizan en el contexto del aula y que implican el uso de tecnologías. Entendemos por actividad didáctica todo aquello que, en el contexto del aula de ciencias, demanda del alumnado algún tipo de acción física o mental cuya finalidad sea que se apropien de algún dominio científico (Cañal, 2000). Nuestra postura es que la efectividad de estas actividades está en función de su potencial para promover ciertos objetivos y contenidos asociados a la didáctica de las ciencias. Aproximarse a lo que estas actividades promueven, permite distinguir entre lo que pueden lograr en principio y lo que realmente se logra con ellas en prácticas situadas.

\section{Metodología y marco de interpretación}

El estudio aquí reportado está centrado en el caso de un profesor de biología de secundaria, a quien Ilamaremos Dante -llamado así con fines de confidencialidad-, cuyos alumnos tienen un promedio de edad de 12 a 13 años. Dante participó inicialmente en un estudio 
que exploró las concepciones de los profesores sobre la ciencia, el aprendizaje y el uso de las tecnologías en la enseñanza de las ciencias (Blancas, 2010). En dicho estudio aplicamos un cuestionario de 18 preguntas que se corresponden a las categorías analíticas establecidas previamente según el ámbito de estudio de nuestro interés (Tabla 1); las categorías de los primeros dos ámbitos -epistemológico y de aprendizaje- fueron retomadas de Rodríguez y López (2006). Las respues- tas dadas al cuestionario fueron categorizadas en tres grandes enfoques: en lo epistemológico (empirismo, racionalismo y constructivismo), en el aprendizaje (asociacionista, cognoscitivista y constructivista) y en lo tecnológico (uso técnico, práctico y crítico). Con esta categorización seleccionamos a Dante, ya que fue el profesor que presentó un marcado perfil conceptual de carácter constructivista en los tres ámbitos de estudio.

Tabla 1

\begin{tabular}{|c|c|c|c|c|c|}
\hline \multicolumn{6}{|c|}{ Categorías de análisis por ámbito de estudio } \\
\hline & Ámbito epistemológico & & Ámbito de aprendizaje & & Ámbito tecnológico \\
\hline 1. & Origen del conocimiento & 1. & En qué consiste el aprendizaje & & Tipo de contenidos \\
\hline 2. & Relación sujeto-objeto & 2. & Papel del sujeto que aprende & 2. & Papel del usuario (alumno) \\
\hline 3. & Método & 3. & Procesos cognitivos & 3. & Tareas implicadas \\
\hline 4. & Correspondencia con la realidad & 4. & Objeto de aprendizaje & 4. & Modalidad de uso \\
\hline 5. & Validación del conocimiento & 5. & Verificación del aprendizaje & 5. & Proceso de comunicación \\
\hline 6. & Finalidad de la ciencia & & Para qué aprender & 6. & Finalidad de uso \\
\hline
\end{tabular}

A Dante, Ingeniero bioquímico con más de 21 años de experiencia docente, le solicitamos elegir algunas actividades de una "experiencia de trabajo" propuesta en ECIT, para que él pudiera realizarlas en clase al abordar un contenido temático. Para ello le entregamos con anticipación dicho material a fin de que lo explorara y decidiera qué actividades realizaría con sus alumnos. Posteriormente, observamos y video-grabamos su práctica en el aula de clase, en el laboratorio escolar y en el aula de medios, lugar donde usualmente los maestros de secundaria llevan a sus alumnos para realizar actividades con tecnología.

Para el trabajo de investigación, tomamos como unidad de análisis cada actividad de ECIT realizada por el profesor. Los hechos observados en la realización de estas actividades se clasificaron a partir de indicadores descriptivos relacionados con las mismas categorías analíticas. De manera conjunta codificamos estos hechos con números de acuerdo al enfoque teórico asociado: para empirismo, asociacionismo y uso técnico, el número 1; para racionalismo, cognoscitivismo y uso práctico, el número 2; para los enfoques relativista, constructivista y uso crítico, el número 3. A partir de la predominancia de estos números valoramos cualitativamente el enfoque que subyace a cada actividad realizada.

\section{Resultados}

Las actividades de ECIT seleccionadas e implementadas por Dante fueron: "Comer para vivir o vivir para comer" y “¿Cómo obtienen sus nutrimentos las plantas?"; que pertenecen a la experiencia de trabajo "Tipos de nutrición" del Bloque II. La primera actividad demanda el uso de un multimedia interactivo, y la segunda el empleo de animaciones en Flash, videos y simulaciones. El análisis aquí presentado está centrado en la segunda de estas actividades porque es la que demanda en mayor medida el uso de tecnología. Una descripción general de esta actividad se muestra en la Tabla 2. 


\begin{tabular}{|c|c|c|}
\hline \multicolumn{3}{|c|}{ Activida d 2. ¿Cómo obtienen sus nutrimentos la s plantas? } \\
\hline Propósitos & \multicolumn{2}{|c|}{$\begin{array}{l}\text {-Identificar que las plantas tien en nutrición autótro fa } \\
\text {-Conocer en qué consiste la foto sintesis. } \\
\text {-Reconocerlos organelos encargados de llevar a cabo la fotosintesis. } \\
\text {-Identificar cuáles son las moléculas que participan y se producen en este proceso. }\end{array}$} \\
\hline $\begin{array}{l}\text { Herramienta } \\
\text { tecnológica }\end{array}$ & \multicolumn{2}{|c|}{$\begin{array}{l}\text {-Animación en flash } \\
\text {-Videos y simulaciones }\end{array}$} \\
\hline \multirow{4}{*}{ Estructura } & Introducción & $\begin{array}{l}\text {-Se presenta a los estudiantes una breve explicación de por qué las } \\
\text { plantas presentan nutrición autótrofa. }\end{array}$ \\
\hline & Exploración & $\begin{array}{l}\text {-Se pide a los alumnos contestar cuatro preguntas que exploran sus } \\
\text { ideas respecto a la nutrición de las plantas. }\end{array}$ \\
\hline & Aplicación & $\begin{array}{l}\text { - Mediante una animación en flash llamada "¿Quién bebe agua por } \\
\text { los pies?", se busca que los estudiantes identifiquen cómo y dónde se } \\
\text { realiza la fotosintesis y que reconozcan la importancia de este } \\
\text { proceso para la vida en el planeta. Se incluyen siete preguntas en } \\
\text { torno a lo presentado en la animacióny que van más allá de ello. } \\
\text {-Después se presenta en video un experimento con el que los } \\
\text { alumnos pueden identificar la producción de oxígeno durante la } \\
\text { fotosintesis. Con las observaciones realizadas en el experimento, se } \\
\text { pide alos alumnos que respondan cinco preguntas. }\end{array}$ \\
\hline & Cierre & $\begin{array}{l}\text {-Se pide a los alumnos responder siete preguntas para dar cierre a la } \\
\text { actividad. }\end{array}$ \\
\hline
\end{tabular}

Nota. Fuente: SEP (2007).

A continuación presentamos el análisis cualitativo de la actividad de ECIT descrita en la Tabla 1, y que fue implementada en el aula por Dante, a partir de cada ámbito de estudio.

\section{Ámbito epistemológico}

Este ámbito alude a la imagen de la ciencia que el profesor comunica, implícita o explícitamente, al realizar la actividad con tecnología. La actividad en el aula estuvo centrada en el recuerdo de información científica y en la observación de fenómenos, más que en generar discusiones teóricas sobre ellos. Dante buscó que los alumnos observaran, describieran y explicaran el fenómeno de la fotosíntesis desde la experiencia sensible. Además, con la actividad promovió que los alumnos comprobaran los conceptos relacionados con la nutrición de las plantas en las animaciones y videos. A su vez, buscó que las respuestas de los alumnos a las preguntas planteadas por la actividad tuvieran correspondencia directa con los conceptos científicos. De acuerdo con las acciones, 4/6 de los indicadores (Tabla 3), se muestra una tendencia de Dante al enfoque empirista de la ciencia en el salón de clase.
Tabla 3

\begin{tabular}{|c|c|}
\hline \multicolumn{2}{|c|}{ Ámbito epistemológico } \\
\hline Indicadores & Enfoque asociado \\
\hline Origen del conocimiento & 1 \\
\hline Relación sujeto-objeto & 1 \\
\hline Método & 2 \\
\hline Correspondencia con la realidad & 2 \\
\hline Validación del conocimiento & 1 \\
\hline Finalidad de la ciencia & 1 \\
\hline Tendencia & 1 \\
\hline
\end{tabular}

Los resultados son contrarios a lo que se espera con la realización de este tipo de actividades que, como recordaremos, están fundamentadas en una visión constructivista. Con ECIT se espera que los alumnos se vean implicados en procesos de construcción de estructuras representacionales y/o modelos teóricos sobre el fenómeno de la fotosíntesis. Al mismo tiempo, se busca que los alumnos tengan diversas formas de acercamiento a éste fenómeno para que las confronten con los modelos teóricos. Sin embargo, en el contexto del aula, lo que importó fue la aprehensión del contenido presentado por la propia actividad y no el acercamiento cualitativo al fenómeno mismo. 


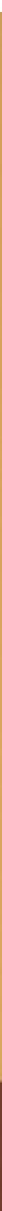


Este ámbito alude a la concepción de aprendizaje que subyace en la realización de la actividad con tecnología. El análisis evidencia una tendencia al enfoque asociacionista del aprendizaje, ya que 4/6 de las acciones son de este tipo (Tabla 4), dado que Dante solicitó constantemente a sus alumnos, de manera oral o escrita, la repetición de información sobre la nutrición de las plantas. Al realizar la actividad, los alumnos tuvieron poca oportunidad de plantear sus ideas, opiniones y/o reflexiones; se dedicaron a responder y a seguir las instrucciones planteadas por la propia actividad o por el profesor. En el aula se promovieron procesos como la mecanización, asociación y repetición de información en torno a la nutrición de las plantas. Con los interactivos, el profesor propició que sus alumnos recordaran información sobre la fotosíntesis y recurrió a éstos para obtener datos que le permitieron dar cuenta de los conceptos que habían adquirido.

\section{Tabla 4}

\begin{tabular}{|c|c|}
\hline \multicolumn{2}{|c|}{ Ámbito de aprendizaje } \\
\hline Indicadores & Enfoque asaciado \\
\hline En qué consiste el aprendizaje & 2 \\
\hline Papel del sujeto que aprende & 1 \\
\hline Procesos cognitivos & 1 \\
\hline Objeto de aprendizaje & 1 \\
\hline Verificación del aprendizaje & 2 \\
\hline Para qué aprender & 1 \\
\hline Tendencia & \\
\hline
\end{tabular}

Los resultados en este ámbito muestran una contrariedad con lo que se espera de la actividad. De manera general, con la actividad se espera que el profesor propicie que los alumnos confronten sus ideas y representaciones respecto a la fotosíntesis; para ello son las animaciones y videos. Sin embargo, esta posibilidad que ofrece la actividad no es aprovechada por Dante, pues con ella solamente buscó demostrar y/o comprobar lo que ocurre en el proceso fotosintético.

\section{Ámbito tecnológico}

Este ámbito alude a las formas en que el profesor usó las herramientas tecnológicas en la realización de la actividad. El análisis mostró una tendencia (5/6 de los indicadores de tipo 1) al enfoque técnico (Tabla 5), ya que Dante usó estas herramientas para transmitir, reafirmar y retroalimentar información sobre la fotosíntesis, y con ello propiciar su memorización. El profesor las utilizó, además, para facilitar el acceso de los estudiantes a contenidos concebidos como verdaderos y absolutos. Los alumnos se dedicaron a seguir mecánicamente las instrucciones de la actividad, sin tener oportunidad de replantearla o adecuarla. Las tareas implicadas propiciaron que los alumnos adquirieran un papel pasivo y, a su vez, los llevaron a conclusiones esperadas por el profesor. Los procesos comunicativos que se configuraron en el aula estuvieron centrados en la transmisión de información.

\section{Tabla 5}

\begin{tabular}{|c|c|}
\hline \multicolumn{2}{|c|}{ Ámbito tecnológico } \\
\hline Indicadores & Enfoque asociado \\
\hline Tipo de contenidos & 1 \\
\hline Papel del usuario (alumnos) & 1 \\
\hline Tareas implicadas & 1 \\
\hline Modalidad de uso & 1 \\
\hline Proceso de comunicación & 1 \\
\hline Finalidad de uso & 2 \\
\hline Tendencia & 1 \\
\hline
\end{tabular}

Los datos en este ámbito muestran que se favorecen ciertos rituales escolares en el aula; como la observación de fenómenos, la obtención, repetición y recuerdo de información y la comprobación de conceptos. Esto se potencia por las propiedades de las herramientas tecnológicas, como la proyección de una animación para exponer un fenómeno natural. Esto es contrario a lo que se espera con ECIT, en donde se sostiene que el empleo de la tecnología propicia procesos que facilitan la construcción de conocimientos de una forma innovadora.

\section{Conclusiones}

Los resultados de nuestro estudio sugieren que un profesor instalado conceptualmente en un enfoque constructivista e implementa actividades con tecnología -que se menciona están fundamentadas en este mismo enfoque-, no garantiza que en el aula de ciencias se configuren procesos pedagógicos de naturaleza cons- 
tructivista. En este estudio de caso resulta interesante cuestionarse cómo Dante, un profesor que se identifica en el plano conceptual con el constructivismo -a través de las respuestas dadas al cuestionario-, al realizar actividades que demandan el uso de la tecnología, regresa a un enfoque de tipo empirista, asociacionista y técnico.

Las propuestas didácticas -como ECIT- representan solo un punto de partida para los procesos de desarrollo y construcción de conocimientos científicos escolares. Por tanto, parece ingenuo pensar que con el hecho de que se diseñen y pongan a disposición del profesorado secuencias didácticas con el uso de la tecnología, habrá un cambio automático en la práctica docente. En el aula, profesores y alumnos reconstruyen estas propuestas e imprimen sentidos y significados a las actividades realizadas. Construir y diseñar actividades con tecnología requiere, sin lugar a dudas, de una comprensión más profunda de los elementos -materiales y contextuales- que inciden en la continua construcción de la cultura que es el aula de ciencias.

En este trabajo sostenemos la postura de que una aproximación cualitativa a las actividades que implican el uso de tecnologías en las aulas, en condiciones naturales y reales, permite reconocer la diversidad de estrategias, conocimientos, saberes y habilidades pedagógicas de los profesores; así como también las distintas finalidades, retos y tensiones de su implementación. Más aún, cuando este tipo de análisis se hace considerando los aspectos que fundamentan la enseñanza de las ciencias, como lo es lo epistemológico, lo cognitivo y lo tecnológico. El hecho de que contemos con evidencias empíricas sobre la forma en que algunos profesores realizan actividades didácticas haciendo uso de tecnologías, es una valiosa oportunidad para apoyarlos a desarrollar, desde la formación docente, modelos didácticos que les permitan ser más conscientes de la intencionalidad, eficacia y posibilidad del uso de dichas herramientas.

Como un punto de partida, y sin pretender agotar el tema, los programas de formación y actualización interesados en apoyar a los profesores de ciencias a incorporar, sistemática y gradualmente, tecnologías en sus prácticas de enseñanza, podrían considerar algunos de los siguientes aspectos:
- Lograr cambios sustanciales a través del uso de las tecnologías que incidan en las prácticas de enseñanza implica que los profesores den sentido al uso de las mismas.

- Reconocer que si bien las tecnologías tienen características reales que posibilitan o amplifican la acción de enseñar, también presentan características que son construidas por los profesores, y que en consecuencia les permiten ejecutar ciertas acciones y no otras.

- Diversificar las herramientas tecnológicas y sus usos en diversas actividades didácticas para explotar sus propiedades y características, así como reconocer sus limitantes y posibilidades de acción.

- Consolidar un conjunto de conocimientos, saberes específicos, habilidades y destrezas profesionales sobre las bases teóricas en que se fundamenta la didáctica de las ciencias.

- Propiciar en los profesores un constante desarroIlo profesional mucho más acorde a las múltiples y diversas demandas de su trabajo.

La preocupación por el uso de tecnologías en la enseñanza de las ciencias debería reflejarse en los procesos de formación y actualización docente, especialmente cuando ésta es una demanda oficial que se disemina a lo largo de diversos documentos y materiales curriculares. Mientras que la atención esté centrada en lo que hemos denominado como un asistencialismo tecnológico y no en lo que cotidianamente ocurre en el aula con el uso de las diversas tecnologías, la formación de los profesores de ciencias, y de otras áreas curriculares, seguirá sin aportar elementos significativos a la ardua e intensa actividad docente. Lo que va a mejorar la enseñanza de las ciencias es una reflexión profunda de sus principales retos y problemas, el diseño y la construcción de alternativas innovadoras para hacer frente a ellos y propuestas mucho más sensibles a los contextos escolares. Hasta el momento no existen tecnologías que puedan lograr esto, lo cual concede razón a la importancia del desarrollo profesional docente para la mejora de la calidad de las prácticas educativas (Vélaz de Medrano y Vailant, 2009). 
Blancas, J. L. (2010). La práctica docente en ambientes tecnológicos para la enseñanza de las ciencias experimentales, a partir de las concepciones de los profesores sobre ciencia aprendizaje y TIC. Tesis de licenciatura. México: UPN.

Cañal, P. (2000). El análisis didáctico de la dinámica del aula: tareas, actividades y estrategias de enseñanza. En: Perales, F. y Cañal, P. (Coord.). Didáctica de las ciencias experimentales: teoría y práctica de la enseñanza de las ciencias, (209-238). España. Editorial Marfil.

Rodríguez-Pineda, D. y López-Mota, A. (2006). ¿Cómo se articulan las concepciones epistemológicas y de aprendizaje con la práctica docente en el aula? Tres estudios de caso de profesores de secundaria. Revista Mexicana de Investigación Educativa, 11 (31), pp. 1307-1335.
Sanmartí, N. e Izquierdo, M. (2001). Cambios y conservación en la enseñanza de las ciencias ante las TIC. Alambique: didáctica de las Ciencias Experimentales, 29, pp. 71-83.

SEP. (2006) Plan de estudios 2006. Educación básica. Secundaria. México: SEP.

SEP. (2006b) Programa de estudio. Asignatura Ciencias, México: SEP. SEP. (2007). Enseñanza de las Ciencias con Tecnología. Software. México: SEP.

Vélaz de Medrano, C. y Vailant, D. (Coord.). (2009). Aprendizaje y desarrollo profesional docente. México: OEI-Fundación Santillana. Obtenido desde http://www.oei.es/publicaciones/ detalle_publicacion. php?id=2)

\section{Diálogo del conocimiento}

El escrito de los maestros Blancas y Rodríguez nos permite aproximarnos a las particularidades que adquieren, en el contexto mexicano, las prácticas de incorporación de las tecnologías de la información y la comunicación en los procesos de enseñanza de las ciencias y a los esfuerzos que realizan los investigadores por profundizar en su estudio y comprensión.

A su vez, nos proporciona un importante referente para contrastar con las experiencia sobre la materia en el caso colombiano y para allegar otros puntos de vista que enriquezcan la indagación en torno a la pertinencia, posibilidades y realizaciones de los educadores colombianos en la apropiación de las tecnologías como medios para propiciar nuevas prácticas de enseñanza de las ciencias.

Por otra parte, el reporte se constituye en un importante ejemplo de las posibilidades y alcances metodológicos que los estudios de caso ofrecen para la comprensión de las prácticas de enseñanza de los maestros de ciencias. Esto permite también situar en su justa medida el papel de los enfoques y las técnicas de investigación cualitativa, muchas veces sobrevalorados en el contexto de la investigación en la enseñanza o en la didáctica de las ciencias.

De paso, el artículo brinda la oportunidad para sopesar las opiniones, no solo antagónicas sino muchas veces infundadas, entre quienes defienden a ultranza el carácter innovador intrínseco y los beneficios a fortiori del uso de las tecnologías en contextos educativos y quienes las descalifican por el solo hecho de su condición de mediadores instrumentales de la racionalidad científico-técnica.

Las conclusiones que se presentan en el escrito ofrecen la oportunidad para decantar juicios y generalizaciones que circulan entre maestros en ejercicio, formadores de maestros e investigadores en enseñanza de las ciencias y en el campo de las tecnologías aplicadas a la educación, en los que suelen primar los compromisos epistemológicos y las convicciones ideológicas de unos y otros acerca del saber tecnológico y sus improntas culturales. Con lo que se subestiman las prácticas culturales que la incorporación intencional y sistemática de dichas tecnologías desencadenan en aulas e instituciones educativas.

Una evidencia adicional de las contradicciones que, con más frecuencia de la esperada, se manifiesta en las prácticas de investigación en educación y del letargo instrumental al que la investigación en enseñanza de las ciencias está permanentemente expuesta como consecuencia de la lógica productiva y del paradigma explicativo que imperan en la comunidad académica.

En este sentido, las recomendaciones que hacen los dos autores, ofrecen también importantes puntos de debate que pueden potenciar el intercambio entre maestros e investigadores en el ámbito latinoamericano. Intercambio que, dicho sea de paso, resulta fundamental para afrontar con criterio y autonomía los retos que la avasallante presencia de las tecnologías en la vida cotidiana imponen a las prácticas atávicas y en muchos casos obsoletas de la institucionalidad educativa, en especial con respecto a la enseñanza de las ciencias.

Juan Carlos Orozco Cruz 